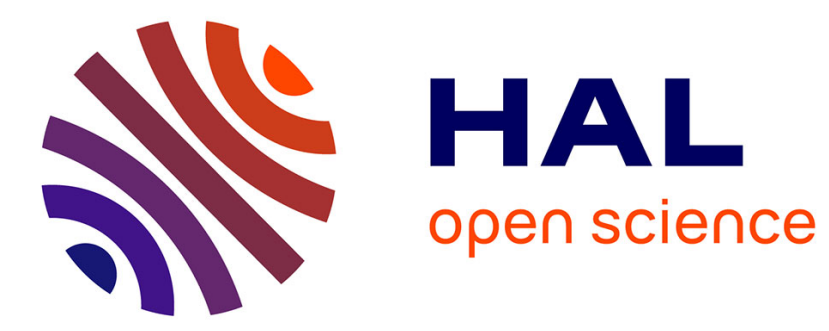

\title{
Pervaporation-induced drying in networks of channels of variable width
}

Kennedy Nexon Chagua Encarnación, Philippe Marmottant, Benjamin Dollet

\section{To cite this version:}

Kennedy Nexon Chagua Encarnación, Philippe Marmottant, Benjamin Dollet. Pervaporation-induced drying in networks of channels of variable width. Microfluidics and Nanofluidics, 2021, 25, pp.71. 10.1007/s10404-021-02467-2 . hal-03321139

\section{HAL Id: hal-03321139 \\ https://hal.science/hal-03321139}

Submitted on 19 Oct 2021

HAL is a multi-disciplinary open access archive for the deposit and dissemination of scientific research documents, whether they are published or not. The documents may come from teaching and research institutions in France or abroad, or from public or private research centers.
L'archive ouverte pluridisciplinaire HAL, est destinée au dépôt et à la diffusion de documents scientifiques de niveau recherche, publiés ou non, émanant des établissements d'enseignement et de recherche français ou étrangers, des laboratoires publics ou privés. 


\title{
Pervaporation-induced drying in networks of channels of variable width
}

\author{
Kennedy Nexon Chagua Encarnación . \\ Philippe Marmottant · Benjamin Dollet
}

Received: date / Accepted: date

\begin{abstract}
We study the influence of variations of cross-section on the drying dynamics by pervaporation in microfluidic channels surrounded by waterpermeable polydimethylsiloxane (PDMS) walls. Single channels of linearly increasing and decreasing width display similar drying time, but different dynamics towards final drying. Single channels with stepwise increasing or decreasing width show jumps of the drying velocity when the meniscus separating the water-filled and the dry part of the channel crosses the steps. In branched networks, narrower branches tend to dry faster than wider ones. All these observations are rationalised in the framework of a model based on the solution of the diffusion equation of water through PDMS, accounting precisely for the local channel geometry, provided the section variation is slow. This model shows that drying velocity depends strongly on the local channel width at the meniscus location. It captures quantitatively our measurements, except the magnitude of the velocity jumps in channels with steps. Implications and perspectives of this study are discussed in the context of leaf drying by the so-called air seeding mechanism in trees.
\end{abstract}

\section{Introduction}

Global warming is an increasing thread for the survival of forests [1,2]. Evapotranspiration at the surface of the leaves, the mechanism whereby sap cir-

K. N. Chagua Encarnación

Univ. Grenoble Alpes, CNRS, LIPhy, 38000 Grenoble, France

Present address: Laboratoire de Physique, ENS Lyon, 46 allée d'Italie, 69364 Lyon Cedex 07, France

P. Marmottant

Univ. Grenoble Alpes, CNRS, LIPhy, 38000 Grenoble, France

B. Dollet

Univ. Grenoble Alpes, CNRS, LIPhy, 38000 Grenoble, France

E-mail: benjamin.dollet@univ-grenoble-alpes.fr 
culates in the xylem from the roots to the leaves, puts the sap in a state of negative pressure $[3,4]$, all the more that the ambient atmospheric conditions are dry and warm. Cavitation bubbles may then appear in the sap network, and subsequently invade it, which can stop the sap circulation. It is therefore of primary importance to understand this invasion mechanism in leaves, known as air seeding among the plant physiologists.

Like the vascular network of animals, leaf venation forms complex networks with different levels of hierarchy, reconnections, and different sections. The channel section tends to decrease in size after each bifurcation, when a channel divides into several channels: the section becomes smaller and smaller until the terminal veins. It was shown the section follows Murray's law as in animal networks [5]: the sum of conduit diameters raised to power 3 is conserved.

During evapotranspiration, water is transported from the veins to the stomata at the surface of the leaves, by diffusion through the mesophyll tissue which surround xylem sap in leaves. This transport is also called pervaporation in other contexts, such as chemical engineering; see e.g. [6-8], and [9] for a full review. To make a biomimetic system mimicking water transport through the mesophyll tissue, we use channels made of polydimethylsiloxane (PDMS), following the method by [10]. This material is permeable to water vapour, like the mesophyll tissues. Among the different microfluidic or nanofluidic systems which have been used for synthetic trees or leaves, soft PDMS was preferred over the stiff hydrogels $[11,12]$ or nanoporous silicon [13], which can withstand negative pressure. Here we do not focus on negative pressure and cavitation, but on latter stages when air invades the network during drying, at pressures close to atmospheric pressure. PDMS has then the advantage that it reproduces better the thinness and flexibility of real leaves.

From a physical standpoint, to understand pervaporation-induced air invasion dynamics in biomimetic networks, we study the simplest systems, increasing the complexity by steps. We started by single channels [14], then elementary networks [15], but still at constant section. In the current study, we release this restriction, and focus on the effect of the variation of section in single channels and simple branched networks.

\section{Materials and methods}

All our channels are molded in a thin PDMS layer glued on a glass plate, as follows. The glass slide was exposed for $30 \mathrm{~s}$ to a plasma, then the PDMS imprint was manually deposited on the plasma-activated glass surface, taking care to avoid folds and blisters of the flexible PDMS sheet. In all this study, the height $h$ of the channel and the thickness $H$ of the PDMS layer (Fig. 1b) are kept as much constant as possible, within the uncertainties of the fabrication process: $h=42 \pm 3 \mu \mathrm{m}$ and $H=88 \pm 2 \mu \mathrm{m}$ (see later Tab. 1 for the values of $h$ and $H$ measured for separate designs). Hence, to study variations of the cross-section, we focus on variations of the channel width $w$ (Fig. 1a). We study first single channels of variable width, then simple networks of channels 
(a)

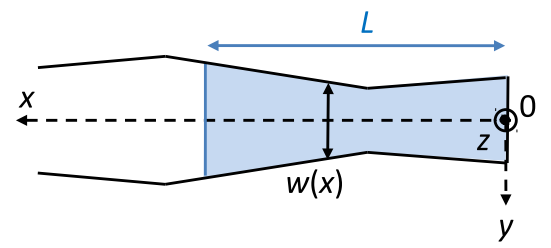

(b)

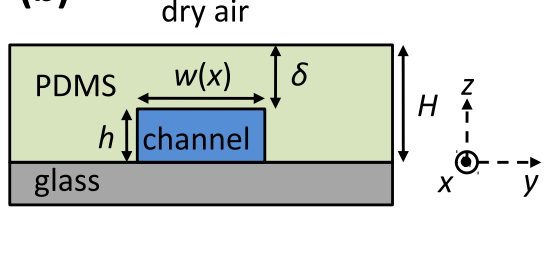

Fig. 1 (a) Top view sketch of a channel of varying width $w(x)$, where $x$ is the streamwise coordinate along its centreline. The channel is filled with water over a length $L$ along its centreline. The origin $x=0$ is located at the closed end of the channel, and the water occupies the region $0 \leq x \leq L$; the meniscus separating the water-filled part of the channel and its air-filled part is thus located at $x=L$. (b) Transverse side view of the channel of width $w(x)$ and height $h$ between a glass slide and a PDMS sheet of thickness $H$.

of different widths. However, for the sake of simplicity, in the latter case, each portion of network between consecutive nodes is of constant width.

The experiments are run as follows. The channels are manually cut with a scalpel at the entrance point (the cuts are clearly visible in Fig. 2). The glass slide on which the channels are bond is plunged into a beaker of deionised water under low pressure for one hour, until the channels are completely filled. They are then placed under a constant flux of dry air, and their drying from the entrance points onwards is recorded with a CCD camera, from which the location of the meniscus (or menisci) separating the water-filled part and airfilled part of the channels is measured by image analysis.

First, we study two single channels of continuously variable width between the entrance point and a terminal wall (Fig. 2a). We focus on channels of total length $L_{c}=12 \mathrm{~mm}$ with width varying linearly along the channel between $50 \mu \mathrm{m}$ and $200 \mu \mathrm{m}$, either decreasing or increasing from the entrance to the terminal wall. Second, we study two single channels of total length $L_{c}=10 \mathrm{~mm}$ with variations of width by steps (Fig. 2b). One channel present five portions (each of length $2 \mathrm{~mm}$ ) with widths decreasing from $175 \mu \mathrm{m}$ to $75 \mu \mathrm{m}$, and the other has four portions (each of length $2.5 \mathrm{~mm}$ ) with widths increasing from $75 \mu \mathrm{m}$ to $150 \mu \mathrm{m}$.

We study next simple channel networks, and we revisit the branched networks which we have studied in [15]. We focus on two configurations. First, we study single-node networks, where an entrance channel splits into two, three, four or five branches (Fig. 2c). In this paper, each of these branches has a different width. Second, we consider a "tree" with consecutive subdivisions of the channels into branches of smaller widths (Fig. 2d). The choice of the geometry of the tree results from a compromise between the presence of certain ingredients of complexity (several levels of branch divisions, branches of different lengths and widths), with yet a simple enough design to allow for simple analysis. 


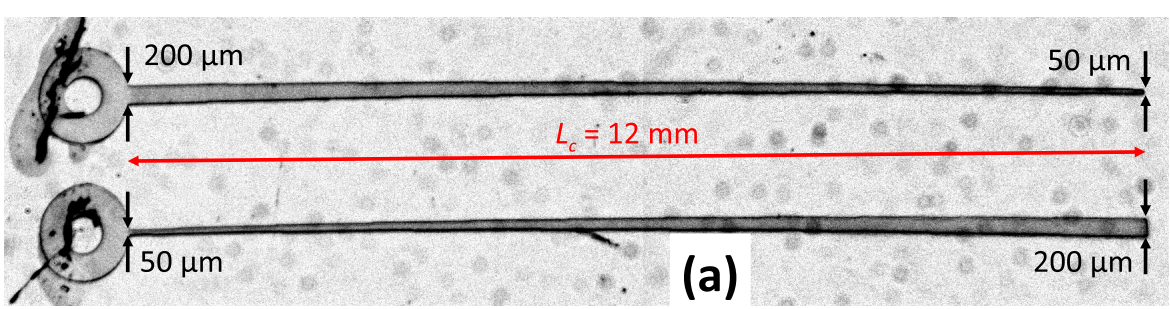

(b)
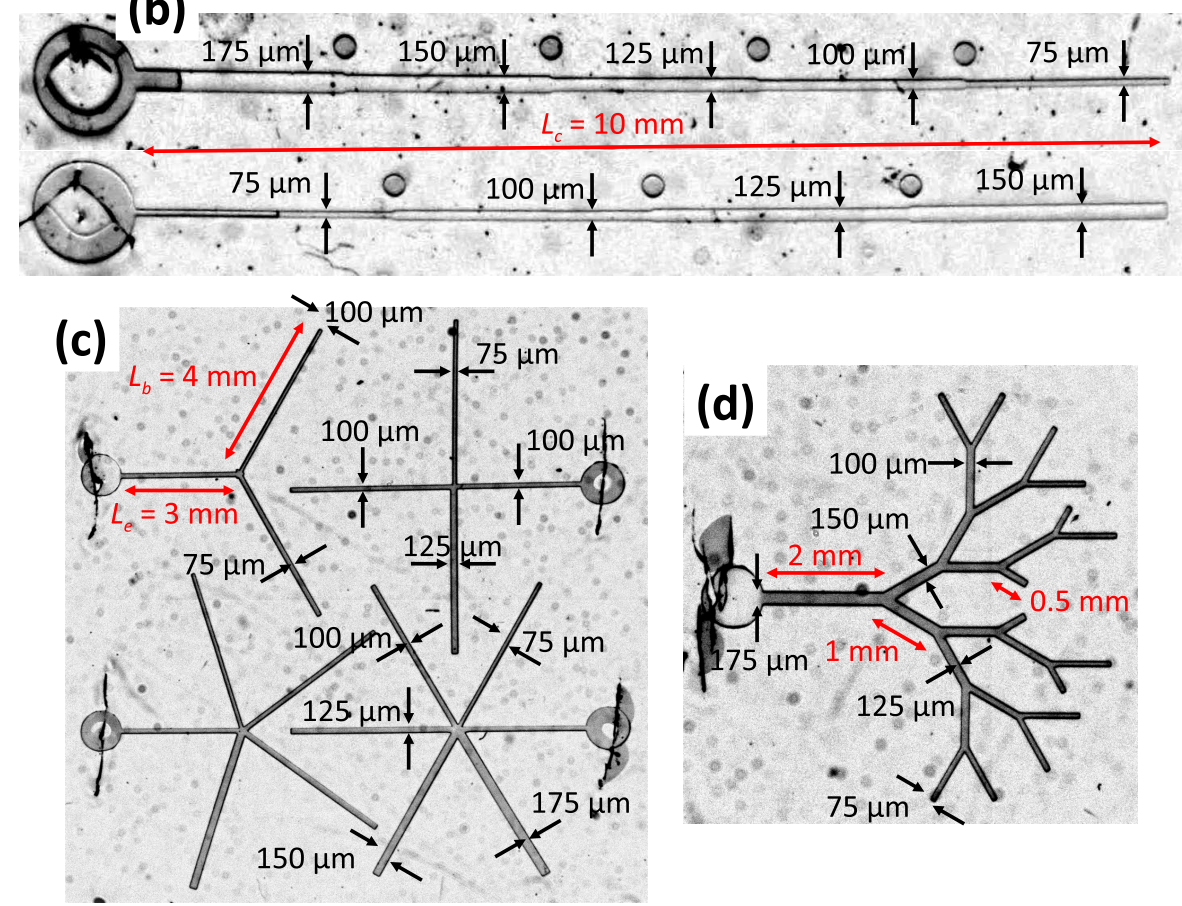

Fig. 2 Snapshots of the channel designs studied in this paper. (a) Single channels of linearly varying width. Notice their slight curvature, due to a slight distortion of the flexible PDMS layer when it is bond to glass; this has no noticeable influence on the dynamics. (b) Single channels with widths varying by steps, which are marked by circles outside the channels. (c) Single-node networks with varying number of branches (the results with the network with four branches will not be presented here). All entrance channels have a width $100 \mu \mathrm{m}$ and a length $L_{e}=3 \mathrm{~mm}$. All branches have a length $L_{b}=4 \mathrm{~mm}$. (d) "Tree" design. Branches have a length of 2,1 or $0.5 \mathrm{~mm}$, and their width decrease at each bifurcation: $175 \mu \mathrm{m}$ for the entrance channel, then 150, 125, 100 and $75 \mu \mathrm{m}$.

\section{Single channels of variable width}

\subsection{Theory}

In this Section, we model the drying dynamics by pervaporation of a single channel of varying width $w(x)$, where $x$ is the streamwise coordinate along the centreline of the channel (Fig. 1a). The channel height is denoted $h$ and 
the PDMS thickness $H$ (Fig. 1b); $\delta=H-h$ is therefore the PDMS thickness between the channel top and the outer air. If $Q$ is the volumetric flux of water leaving the water-filled part of the channel, the conservation of mass writes:

$$
-Q=\frac{\mathrm{d}}{\mathrm{d} t}\left[h \int_{0}^{L(t)} w(x) \mathrm{d} x\right]=h \dot{L} w(x=L),
$$

where the term in brackets is the volume of water inside the channel at time $t, L$ being the length of the water-filled part (henceforth called water length) and $|\dot{L}|$ the velocity of the meniscus. It is worth noting that at a given flux $Q$, the larger the width $w(x=L)$ at the location of the meniscus, the slower the meniscus.

There are two contributions to the flux $Q=Q_{\ell}+Q_{g}$ : one by diffusion from the water-PDMS interface $\left(Q_{\ell}\right)$, and an evaporative flux $Q_{g}$ at the meniscus. We restrict our analysis to a slowly varying width: $|\mathrm{d} w / \mathrm{d} x| \ll 1$. This hypothesis is suitable for slender channels, such as those encountered in leaves. For a slowly varying width, the streamwise dependence of the concentration field of water into PDMS is much weaker than the spanwise dependence, and we may neglect the width variation to estimate the diffusive flux. Hence:

$$
Q_{\ell}=\int_{0}^{L} q_{\ell}(w(x)) \mathrm{d} x,
$$

where $q_{\ell}(w)$ is the diffusive flux per unit length of a channel of constant width $w$. Inserting in (1), we get:

$$
h \dot{L} w(x=L)=-\int_{0}^{L} q_{\ell}(w(x)) \mathrm{d} x-Q_{g} .
$$

In [14], we have derived the following analytical predictions of the flux $q_{\ell}$ :

$$
q_{\ell}=D_{P} \bar{c}_{P}^{\mathrm{sat}}(1-\mathrm{RH}) \tilde{q}_{\ell},
$$

with $D_{P}=10^{-9} \mathrm{~m}^{2} / \mathrm{s}$ the diffusivity of water in PDMS [18], $\bar{c}_{P}^{\text {sat }}=M C_{P}^{\text {sat }} / \rho$ the mass fraction of water in PDMS at saturation (with $M=0.018 \mathrm{~kg} / \mathrm{mol}$ the molar mass of water, $\rho=10^{3} \mathrm{~kg} / \mathrm{m}^{3}$ its density, and $C_{P}^{\text {sat }}=40 \mathrm{~mol} / \mathrm{m}^{3}$ the saturation concentration of water in PDMS [17], this gives $\bar{c}_{P}^{\text {sat }}=7.2 \times 10^{-4}$ ), $\mathrm{RH}$ the relative humidity of the outer air (henceforth set to zero, consistently with our experiments) and $\tilde{q}_{\ell}$ a dimensionless shape factor coming from the resolution of a diffusion problem:

$$
\tilde{q}_{\ell}=\frac{w}{\delta}+\xi
$$

with $\xi$ another dimensionless shape factor quantifying diffusion between the channel side walls and the outer air; under reasonable geometrical assumptions quantified in [14] and holding in our experiments, it equals:

$$
\xi=\frac{2}{\pi}\left[\ln \frac{(H+\delta) h}{\delta^{2}}+\frac{H}{\delta} \ln \frac{H+\delta}{h}\right] .
$$


We also use the prediction of $Q_{g}$ made in [14] with the local value of the thickness at the meniscus location, simply denoted $w$ in the remainder of this Section. To be rigorous, the assumption $|\mathrm{d} w / \mathrm{d} x| \ll 1$ is not sufficient to make such an approximation, and should be replaced by the more stringent condition $|\mathrm{d} w / \mathrm{d} x| \ll \varepsilon^{1 / 2}$ where $\varepsilon=D_{P} / \alpha D_{a}$ is a small parameter (with $\alpha=0.03$ the Henry constant quantifying the water affinity in PDMS [16] and $D_{a}=2 \times 10^{-5} \mathrm{~m}^{2} / \mathrm{s}$ the diffusivity of water vapour in air, hence $\varepsilon=2 \times 10^{-3}$ ), because the value of $Q_{g}$ depends on the concentration field of water at distances of order $\varepsilon^{-1 / 2} w$ away from the meniscus [14]. Under this approximation, the following prediction holds:

$$
Q_{g}=\sqrt{\alpha D_{a} D_{P} h w \tilde{q}_{\ell}}
$$

\subsection{Experiments}

\subsubsection{Channels with linearly variable width}

We now test our theory on the two channels with linearly variable width. Fig. 3a shows the time evolution of the water length in these two channels. It is a decreasing function of time, consistently with the drying process. More interestingly, both curves are convex, but the convexity is larger for the channel with increasing width compared with the one with decreasing width. The meniscus in the first channel is faster at the beginning of the drying process, but slower at the end, and the total drying time is almost similar for both channels.

Qualitatively, this behaviour highlights the influence of the local value of the channel width at the meniscus location in Eq. (1): the narrower the channel, the faster the meniscus at given flux $Q$. Quantitatively, we can apply the model of Sec. 3.1 for a width profile of the form:

$$
w(x)=w_{0}+\left(w_{1}-w_{0}\right) \frac{L_{c}-L-x}{L_{c}},
$$

with $L_{c}$ the channel length, $w_{0}$ the width at the channel entrance and $w_{1}$ the width at its closed end. Inserting (7) in (2) and using (4) and (5), we get:

$$
Q_{\ell}=D_{P} \bar{c}_{P}^{\mathrm{sat}}\left[\left(\frac{w_{1}}{\delta}+\xi\right) L-\frac{w_{1}-w_{0}}{2 \delta L_{c}} L^{2}\right]
$$

Using the expression (6) of $Q_{g}$, we get after some algebra:

$$
\begin{aligned}
{\left[1-\left(1-\frac{w_{0}}{w_{1}}\right) \bar{L}\right] \frac{\mathrm{d} \bar{L}}{\mathrm{~d} t}=} & -\frac{D_{1}}{h w_{1}}\left[\left(\frac{w_{1}}{\delta}+\xi\right) \bar{L}+\frac{w_{1}-w_{0}}{2 \delta} \bar{L}^{2}\right]-\frac{D_{2}}{L_{c} \sqrt{h \delta}} \\
& \times \sqrt{\left(1+\frac{w_{0}-w_{1}}{w_{1}} \bar{L}\right)\left(1+\frac{w_{0}-w_{1}}{w_{1}} \bar{L}+\frac{\delta}{w_{1}} \xi\right)(8)}
\end{aligned}
$$




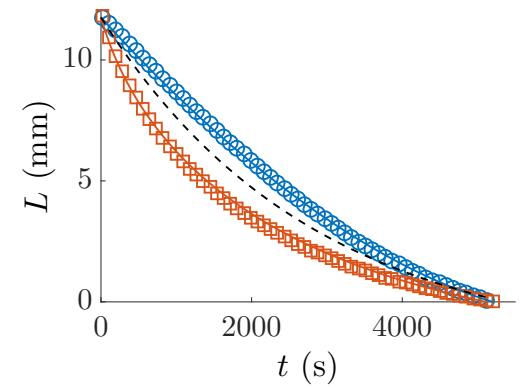

(a)

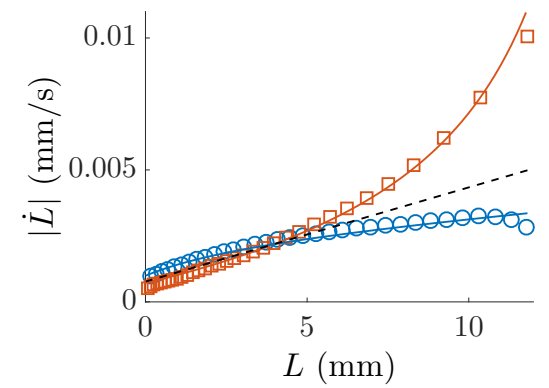

(b)

Fig. 3 Drying dynamics in channels of width varying linearly between 50 and $200 \mu \mathrm{m}$, for a decrease (o) and an increase $(\square)$ of the width over a length of $12 \mathrm{~mm}$. (a) Time evolution of the water length, and (b) velocity of the meniscus as a function of the water length. In (b), the plain curves are fits by Eq. (8) with $D_{1}$ and $D_{2}$ as fitting parameters. In (a), the plain curves result from numerical integration of the fitting curves of (b). The dotted curves show the case of a channel of constant width $125 \mu \mathrm{m}$.

with the dimensionless quantity: $\bar{L}=L / L_{c}$, and two physicochemical parameters having the dimension of a diffusivity:

$$
D_{1}=D_{P} \bar{c}_{P}^{\mathrm{sat}}, \quad D_{2}=\sqrt{\alpha D_{a} D_{P}} .
$$

Eq. (8) provides an explicit relationship between the instantaneous velocity of the meniscus and its location. Therefore, to compare our data and our model, we plot the velocity of the meniscus as a function of the water length in Fig. 3b, and we fit the curves by (8) with $D_{1}$ and $D_{2}$ as fitting parameters. We anticipate that these two parameters will serve as fitting parameters for each set of experiments to be discussed throughout this study, and we refer to Sec. 5 for a discussion of their values.

Fig. 3 shows that the fit is excellent. Only small discrepancies are visible for largest values of $L$, which corresponds to cases where the meniscus is close to the open end of the channel and may be influenced by the uncontrolled opening of the channel. Furthermore, if we fit independently the data for each channel, the values of the fitting parameters are in close agreement (see later Tab. 1). This shows the accuracy of our model to explain data in channels of slowly varying width.

\subsubsection{Channels with stepwise varying width}

We proceed with two channels of stepwise varying length. Fig. 4a shows the time evolution of the water length in these two channels. It is once again a decreasing function of time. However, the novel feature of the curves is the occurrence of finite changes of velocity at each step, which shows more clearly when we plot the velocity of the meniscus as a function of the water length (Fig. 4b). The velocity jump is positive, respectively negative, at the steps where the width decreases, respectively increases. 


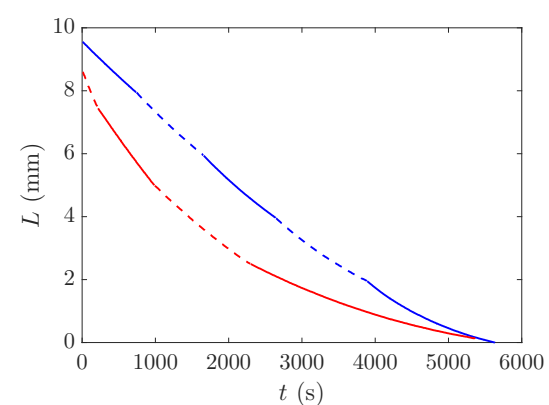

(a)

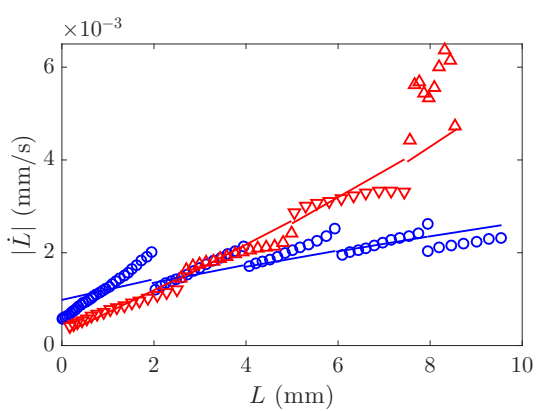

(b)

Fig. 4 Drying dynamics in channels of stepwise varying width. (a) Time evolution of the water length. Here, we use all available data points, which are close enough to constitute a seemingly continuous curve. The top curve corresponds to a decreasing width, and the bottom one to an increasing width. Successive portions of constant width are alternatively figured in plain and dotted lines. (b) Velocity of the meniscus as a function of the water length in channel of stepwise increasing (triangles) or decreasing (circles) width. The lines are fits by Eq. (10) with $D_{1}$ and $D_{2}$ as fitting parameters.

Qualitatively, as discussed in Sec. 3.2.1, this behaviour is consistent with the influence of the local value of the channel width at the meniscus location in (1). We can compare the experimental data with our model, bearing in mind that strictly speaking, the latter does not apply to stepwise variations in width, because it relies on an hypothesis of slowly varying width. We assume that the meniscus is in a portion of width $w_{0}$ and at a distance $L_{0}(t)$ from the upcoming step, and that the water-filled part of the channel spans $N$ full portions of length $L_{i}$ and constant width $w_{i}$ (for $i$ ranging from 1 to $N$ ). The total water length is then $L(t)=L_{0}(t)+\sum_{i=1}^{N} L_{i}$, and the total diffusive flux (2) writes:

$$
Q_{\ell}=q_{\ell}\left(w_{0}\right) L_{0}+\sum_{i=1}^{N} q_{\ell}\left(w_{i}\right) L_{i}=D_{P} \bar{c}_{P}^{\mathrm{sat}}\left[\left(\frac{w_{0}}{\delta}+\xi\right) L_{0}+\sum_{i=1}^{N}\left(\frac{w_{i}}{\delta}+\xi\right) L_{i}\right]
$$

where (4) and (5) have been used. Inserting into (3), we get:

$$
\dot{L}=-\frac{L+L_{\text {sup }}}{\tau},
$$

with:

$$
\tau=\frac{1}{D_{1} A}
$$

and:

$$
L_{\text {sup }}=\frac{D_{2}}{D_{1} \sqrt{A}}-\sum_{i=1}^{N} \frac{w_{i}-w_{0}}{w_{0}+\xi \delta} L_{i},
$$

where $A$ is a channel-dependent geometrical parameter:

$$
A=\frac{1}{h w_{0}}\left(\frac{w_{0}}{\delta}+\xi\right),
$$


and $D_{1}$ and $D_{2}$ are given by (9).

We fit the data of Fig. 4b by Eq. (10) with $D_{1}$ and $D_{2}$ as fitting parameters. Contrary to the channels with linearly variable width, the agreement between the model and the data is not very good; in particular, the model predicts jumps in velocity which are much smaller in amplitude than what is experimentally observed. Possible reasons for this discrepancy are discussed in Sec. 5.2.

\section{Branched channels with different widths}

To extend further our study, we now consider branched channels, yet without reconnections, and with different widths. For simplicity, we study only channels where individual branches have constant widths.

\subsection{Theory}

Let us first consider a meniscus in the entrance channel. It is in contact with a water region which spans part of the entrance channel and all branches. All these contribute to the diffusive flux $Q_{\ell}$ introduced in Sec. 3.1. Hence, Eq. (3) is replaced by:

$$
h \dot{L} w_{m}=-\int q_{\ell}(w(s)) \mathrm{d} s-Q_{g},
$$

where $L$ is the total length of the water region, $w_{m}$ is the value of the width at the location of the meniscus, and the integral on the right-hand side spans all the water region, with $s$ a curvilinear coordinate along the centreline of that region.

Let us specify the analysis to the case relevant to the experiments, of $N$ branches of length $L_{i}$ and constant width $w_{i}$ (for $i$ ranging from 1 to $N$ ) extending from the first node. We also consider that the entrance channel has a constant width $w_{0}$, and that the water inside this channel spans a length $L_{0}(t)$. The total water length is then $L(t)=L_{0}(t)+\sum_{i=1}^{N} L_{i}$, and (13) becomes:

$$
h \dot{L}_{0} w_{0}=-q_{\ell}\left(w_{0}\right) L_{0}-\sum_{i=1}^{N} q_{\ell}\left(w_{i}\right) L_{i}-Q_{g} .
$$

Proceeding in a similar fashion as in Sec. 3.2.2, we obtain that the water length should obey Eq. (10), where the sum extends over all the branches ahead of the meniscus. The dynamics in intermediate channels also follows (14) and thus (10), provided $w_{0}$ is taken as the local width at the meniscus location, and the sum runs over all water-filled branches ahead of the meniscus. The dynamics in terminal branches is obtained by suppressing the sum from (10). In the current case where all branches have a constant width, the dynamics is therefore of the form $\dot{L}_{0}=-\left(L_{0}+L_{\text {sup }}\right) / \tau$, where the characteristic time 
scale $\tau$ depends only on the local width (where the meniscus is located), while the length scale $L_{\text {sup }}$ depends on the whole network ahead of the meniscus.

We proved in [15] that this analysis is true as long as all branches are slender (of length much larger than the transverse dimensions) and well separated (of mutual distances much larger than the transverse dimensions), in order to neglect diffusive coupling between channels separated by a small distance [10].

\subsection{Experiments}

\subsubsection{Single-node networks}

Fig. 5a, c and e shows the distance travelled by the menisci as a function of time in the single-node networks with respectively two, three and five branches. In the entrance channel, the meniscus velocity increases as the number of branches increases, and there is a velocity jump as the meniscus in the entrance channel splits into several menisci in the branches. This result was already found for single-node networks with uniform width, and can be ascribed to a jump of connected water length when meniscus splitting occurs at the node [15]. The new feature here is that the dynamics of each branch depends on its width: the smaller the latter, the larger the meniscus velocity and the shorter the drying time. Qualitatively, this is a signature of the fact that the typical drying time $\tau$ is an increasing function of the width. This can be seen from Eqs. (11) and (12), which show that such a dependence on width arises from the interplay between the diffusion between the channel top wall and the outer air [giving the term $w / \delta$ in (12)], and the diffusion between the channel side walls and the outer air [giving the term $\xi$ in (12)]. We showed in [14] that this interplay is responsible for the faster drying of narrow channels because they are more prone to lose water from their side walls, compared to wider channels.

To test more quantitatively the predictions of Sec. 4.1, we plot the velocity of the meniscus as a function of the water length in the right panel of Fig. 5, and we fit the curves by (10). For each single-node network, we impose that $D_{1}$ and $D_{2}$ be the same for the entrance channel and all the branches. The right panel of Fig. 5 shows that the fit is satisfactory; in particular, the terminal branches are correctly described, also the experimental data show some concavity, which may be ascribed to evaporative coupling effects discussed in [15]. The entrance channels are also well fitted, with the exception of the five-branch network where the experimental slope is too large.

\subsubsection{Tree network}

We now turn to the tree network of Fig. 2d. It comprises 27 branches, but some of them share the same dimensions and "order" in the tree hierarchy, and it is found that they then follow a similar dynamics. We gather such branches into 9 different groups, represented by different symbols in Fig. 6a. 


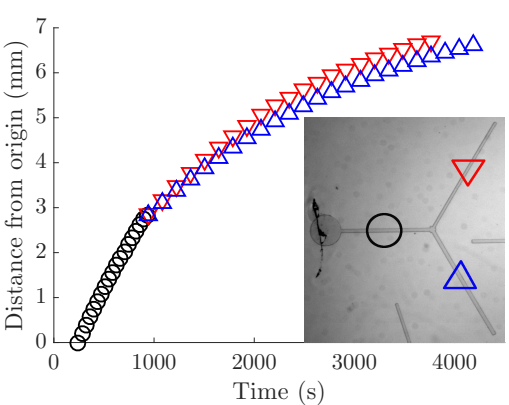

(a)

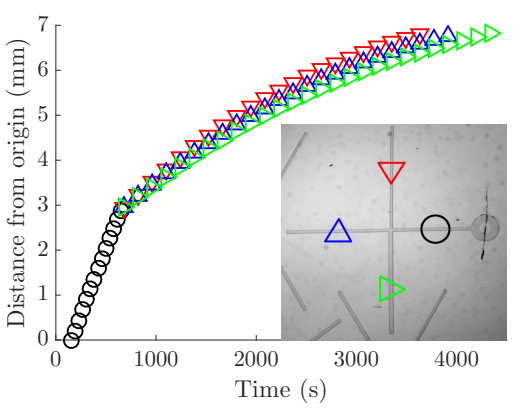

(c)

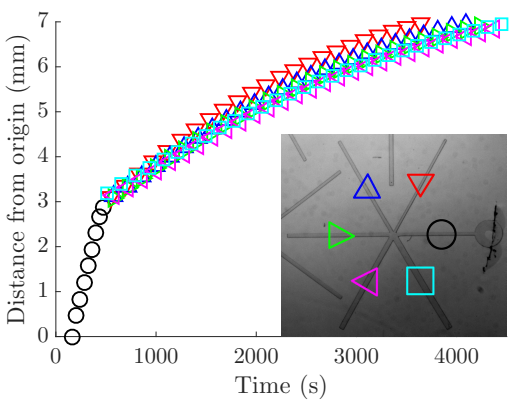

(e)

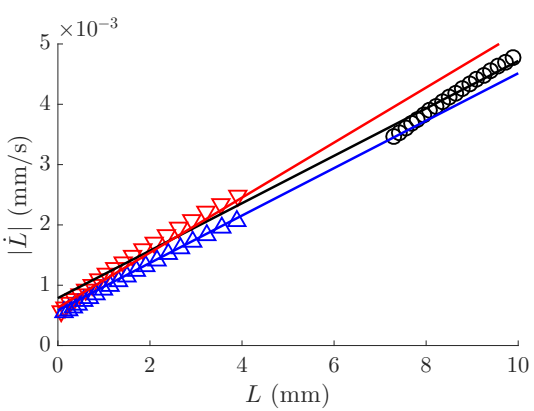

(b)

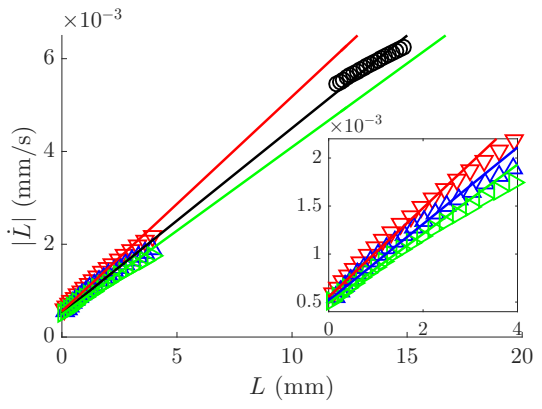

(d)

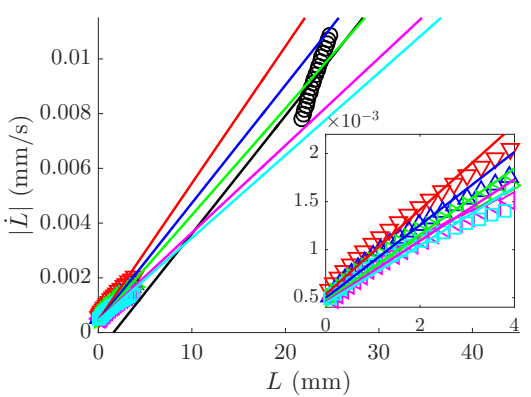

(f)

Fig. 5 Drying dynamics in single-node networks. From bottom to top, networks with two, three and five branches. Left column: temporal evolution of the distance travelled by the menisci from the entrance point. Right column: velocity as a function of the water length. Insets in panels (d) and (f) are zooms on the terminal branches. Symbols: entrance channel $(\circ)$, branches of width $75(\nabla), 100(\triangle), 125(\triangleright), 150(\triangleleft)$ and $175 \mu \mathrm{m}(\square)$. The lines are fits by (10), with $D_{1}$ and $D_{2}$ as fitting parameters.

Fig. $6 \mathrm{~b}$ shows the distance travelled by the menisci as a function of time in the tree, for all branches. Like for the single-node networks, velocity jumps occur at each node. The new feature here is that at given depth in the tree network, the velocity is an increasing function of the water length ahead of the 


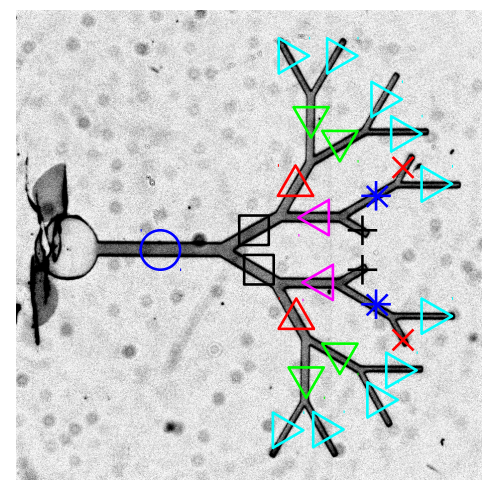

(a)

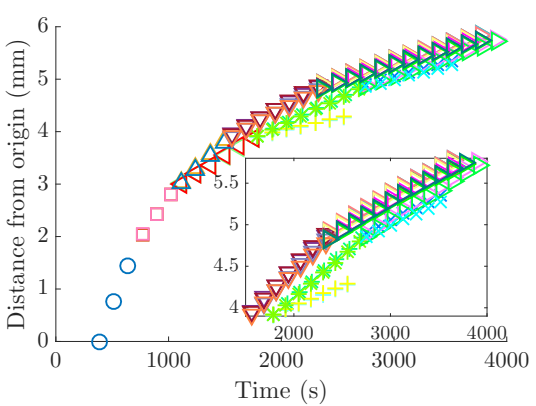

(b)

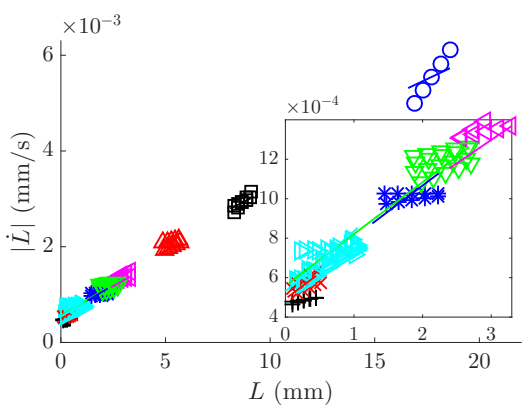

(c)

Fig. 6 Drying dynamics in a tree. (a) Symbols used for the different branches. (b) Temporal evolution of the distance travelled by the menisci from the entrance point. Right column: velocity as a function of the water length. The inset is a zoom on the terminal branches, to help distinguishing their drying dynamics. (c) Velocity as a function of the water length. The lines are fits by (10), with $D_{1}$ and $D_{2}$ as fitting parameters. The inset is a zoom on the bottom left part of the main plot.

considered meniscus: the shorter the branches, the slower the dynamics (see inset of Fig. 6b).

Here again, we plot the velocity of the meniscus as a function of the water length (Fig. 6c), and we fit the curves by (10). We find once again a good agreement between the experiments and the data, which suggests that our physical modelling remains adapted to the case of more complex branched networks.

\section{Discussion}

5.1 Values of the fitting parameters

In each experiment, the two physicochemical parameters $D_{1}=D_{P} C_{P}^{\text {sat }}$ and $D_{2}=\sqrt{\alpha D_{a} D_{P}}$ have been used as fitting parameters. Their values are re- 


\begin{tabular}{|c|c|c|c|c|c|}
\hline Experiment & Figure & $\begin{array}{c}D_{1} \\
\left(\mu \mathrm{m}^{2} / s\right)\end{array}$ & $\begin{array}{c}D_{2} \\
\left(\times 10^{4} \mu \mathrm{m}^{2} / s\right)\end{array}$ & $\begin{array}{c}h \\
(\mu \mathrm{m})\end{array}$ & $\begin{array}{c}H \\
(\mu \mathrm{m})\end{array}$ \\
\hline linearly decreasing width & 3 & 0.410 & 2.53 & 39.3 & 89.3 \\
linearly increasing width & & 0.394 & 2.42 & & \\
\hline stepwise decreasing width & 4 & 0.184 & 2.83 & 45.6 & 84.5 \\
stepwise increasing width & & 0.540 & 0.79 & & \\
\hline 2-branch network & $5 \mathrm{a}, \mathrm{b}$ & 0.389 & 1.83 & & \\
3-branch network & $5 \mathrm{c}, \mathrm{d}$ & 0.395 & 1.62 & 42.2 & 87.4 \\
5-branch network & $5 \mathrm{e}, \mathrm{f}$ & 0.437 & 1.32 & & \\
\hline tree & 6 & 0.265 & 1.57 & 41.4 & 89.2 \\
\hline
\end{tabular}

Table 1 Values of the fitting parameters $D_{1}$ and $D_{2}$, and of the dimensions $h$ and $H$, for all experiments.

ported in Tab. 1. They show some dispersion, especially for the channels with stepwise varying width, for which it has been shown that the model is inaccurate. The other experiments yield $D_{1}=0.4 \mu \mathrm{m}^{2} / \mathrm{s}$ and $D_{2}=2 \times 10^{4} \mu \mathrm{m}^{2} / \mathrm{s}$. Taking values from the literature: $D_{P}=10^{-9} \mathrm{~m}^{2} / \mathrm{s}[18], D_{a}=2 \times 10^{-5} \mathrm{~m}^{2} / \mathrm{s}$, $\alpha=0.03[16]$ and $\bar{c}_{P}^{\text {sat }}=M C_{P}^{\text {sat }} / \rho=7.2 \times 10^{-4}$ (using in particular $C_{P}^{\text {sat }}=$ $40 \mathrm{~mol} / \mathrm{m}^{-3}[17]$ ), we compute $D_{1}=0.7 \mu \mathrm{m}^{2} / \mathrm{s}$ and $D_{2}=2 \times 10^{4} \mu \mathrm{m}^{2} / \mathrm{s}$. Bearing in mind that the values of the physicochemical parameters $D_{P}, \alpha$ and $C_{P}^{\text {sat }}$ are known with relatively poor precision, we can conclude that the values of our fitting parameters are in good agreement with the reported values of $D_{P}, \alpha$ and $C_{P}^{\text {sat }}$.

\subsection{Limitations of the current model and perspectives}

We have compared all our experiments with a model based on the hypothesis of slowly varying width. This enables to consider that water diffusion through PDMS acts mainly in the transverse direction. We have shown that such a model captures well our experimental observations, except the case of channels with stepwise varying width.

Obviously, in such a case, the hypothesis of slowly varying width is violated at the location of the steps. To go beyond and extend the model, the full 3D diffusion of water through PDMS should be solved, at least in the vicinity of the steps. However, this requires time-consuming numerical simulations, beyond the scope of this study.

Moreover, an additional ingredient may become important in the case of steps: when passing an abrupt variation in channel section, the meniscus curvature necessarily varies at given contact angle, to match the geometry of the channel walls. In other contexts, such an effect is important for droplet breakup in microfluidic T-junctions [19] and in soap films and foams in porous media [20-22]. This induces variations of capillary pressure, hence of pressure in the liquid, which may deform the channel, an effect hitherto disregarded in the model. This might explain why velocity jumps in Fig. 4 are much more marked in experiments that in the model. Interestingly, in the context of tree physiology, such effects are relevant in the xylem network of trees $[23,24]$, 
where xylem vessels are separated by pits of nanometric dimensions where menisci between embolism and liquid can get trapped while undergoing huge variations of capillary pressure. In an upcoming study, we will revisit this effect by mimicking pits in microfluidic experimental micromodels, to clarify the couplings between drying, meniscus dynamics, capillary pressure variations and channel deformability.

\section{Conclusions}

Our study revealed that drying in networks with channels of variable sections are well described by a generalised version of our model, consisting in the integration of the section-dependent diffusive flux over the length of the channels (Eq. 3). This model fits very well with experiments for linearly varying section, stepwise-decreasing width and division in branches with different sections. The drying dynamics is always smooth, without any arrest, just velocity changes. However, studies on real leaves $[25,26]$ showed a stop-and-go dynamics with arrests and sudden jumps. This peculiar dynamics may be the consequence of the fact that conduits are not fully continuous in plants, but are made of collection of separate conduits connected by pits. Future work will focus on the effect of tiny constrictions in drying dynamics.

Acknowledgements We thank Aurélien Caumont, Danièle Centanni and Ludovic Keiser for experimental help. Funding by ANR, under the grant PHYSAP ANR619-CE30-0010-02, is gratefully acknowledged.

\section{Conflict of interest}

The authors declare that they have no conflict of interest.

\section{References}

1. B. Choat, T. J. Brodribb, C. R. Brodersen, R. A. Duursma, R. López, B. E. Medlyn, Triggers of tree mortality under drought, Nature, 558, 531-539 (2018).

2. T. J. Brodribb, J. Powers, H. Cochard, B. Choat, Hanging by a thread? Forests and drought, Science, 368, 261-266 (2020).

3. A. D. Stroock, V. V. Pagay, M. A. Zwieniecki, N. M. Holbrook, The physicochemical hydrodynamics of vascular plants, Annu. Rev. Fluid Mech., 46, 615-642 (2014).

4. K. H. Jensen, K. Berg-Sørensen, H. Bruus, N. M. Holbrook, J. Liesche, A. Schulz, M. A. Zwieniecki, T. Bohr, Sap flow and sugar transport in plants, Rev. Mod. Phys, 88, 035007 (2016).

5. K. A. McCulloh, J. S. Sperry, F. R. Adler, Water transport in plants obeys Murray's law, Nature, 421, 939-942 (2003).

6. J. C. T. Eijkel, J. G. Bomer, A. van den Berg, Osmosis and pervaporation in polyimide submicron microfluidic channel structures, Appl. Phys. Lett., 87, 114103 (2005).

7. N. Ziane, M. Guirardel, J. Leng, J. B. Salmon, Drying with no concentration gradient in large microfluidic droplets, Soft Matter, 11, 3637-3642 (2015).

8. I. Ziemecka, B. Haut, B. Scheid, Hydrogen peroxide concentration by pervaporation of a ternary liquid solution in microfluidics, Lab Chip, 15, 504-511 (2015). 
9. P. Bacchin, J. Leng, J. B. Salmon, Microfluidic evaporation, pervaporation and osmosis: from passive pumping to solute concentration, under review in Chem. Rev.

10. X. Noblin, L. Mahadevan, I. A. Coomaraswamy, D. A. Weitz, N. M. Holbrook, M. A. Zwieniecki, Proc. Natl. Acad. Sci., 105, 9140-9144 (2008).

11. T. D. Wheeler, A. D. Stroock, The transpiration of water at negative pressures in a synthetic tree, Nature, 455, 208-212 (2008).

12. O. Vincent, P. Marmottant, P. A. Quinto-Su, C. D. Ohl, Birth and growth of cavitation bubbles within water under tension confined in a simple synthetic tree, Phys. Rev. Lett., 108, 184502 (2012).

13. O. Vincent, P. Marmottant, S. R. Gonzalez-Avila, K. Ando, C. D. Ohl, The fast dynamics of cavitation bubbles within water confined in elastic solids, Soft Matter, 10, 1455-1461 (2014).

14. B. Dollet, J. F. Louf, M. Alonzo, K. H. Jensen, P. Marmottant, Drying of channels by evaporation through a permeable medium, J. R. Soc. Interface, 16, 20180690 (2019).

15. B. Dollet, K. N. Chagua Encarnación, R. Gautier, P. Marmottant, Drying by pervaporation in elementary channel networks, J. Fluid Mech., 906, A6 (2021).

16. S. J. Harley, E. A. Glascoe, R. S. Maxwell, Thermodynamic study in dynamic water vapor sorption in Sylgard-184, J. Phys. Chem. B, 116, 14183-14190 (2012).

17. G. C. Randall, P. S. Doyle, Permeation-driven flow in poly(dimethylsiloxane) microfluidic devices, Proc. Natl. Acad. Sci., 102, 10813-10818 (2005).

18. J. M. Watson, M. G. Baron, The behaviour in water in poly(dimethylsiloxane), J. Membr. Sci., 110, 47-57 (1996).

19. C. P. Ody, C. N. Baroud, E. de Langre, Transport of wetting liquid plugs in bifurcating microfluidic channels, J. Colloid Interface Sci., 308, 231-238 (2007).

20. Q. Xu, W. R. Rossen, Effective viscosity of foam in periodically constricted tubes, Colloids Surf. A, 216, 175-194 (2003)

21. S. J. Cox, S. Neethling, W. R. Rossen, W. Schleifenbaum, P. Schmidt-Wellenburg, J. J. Cilliers, A theory of the effective yield stress of foam in porous media: the motion of a soap film traversing a three-dimensional pore, Colloids Surf. A, 245, 143-151 (2004).

22. B. Géraud, Y. Méheust, I. Cantat, B. Dollet, Lamella division in a foam flowing through a two-dimensional porous medium: A model fragmentation process, Phys. Rev. Lett., 118, 098003 (2017).

23. M. T. Tyree, J. S. Sperry, Vulnerability of xylem to cavitation and embolism, Annu. Rev. Plant Physiol. Plant Mol. Biol., 40, 1936 (1989).

24. B. Choat, M. Ball, J. Luly, J. Holtum, Pit membrane porosity and water stress-induced cavitation in four co-existing dry rainforest tree species, Plant Physiol., 131, 41-48 (2003).

25. A. Ponomarenko, O. Vincent, A. Pietriga, H. Cochard, E. Badel, P. Marmottant, Ultrasonic emissions reveal individual cavitation bubbles in water-stressed wood, J. R. Soc. Interface, 11, 20140480 (2014)

26. T. J. Brodribb, D. Bienaimé, P. Marmottant, Revealing catastrophic failure of leaf networks under stress, Proc. Natl. Acad. Sci., 113, 4865-4869 (2016). 